\title{
Other Asian
}

National Cancer Institute

\section{Source}

National Cancer Institute. Other Asian. NCI Thesaurus. Code C107601.

A person having origins in the original peoples of Asia, but not China, India, Korea, the Philippine Islands or Vietnam. 